\title{
DIE BEDEUTUNG DES INSTRUMENTALEN SPIELS IM HÖLLENAKT AUS RAMEAUS CASTOR ET POLLUX
}

\author{
THE IMPORTANCE OF INSTRUMENTAL PLAYING \\ IN THE HELL ACT FROM RAMEAU'S CASTOR ET POLLUX
}

\section{LA IMPORTANCIA DE LA MÚSICA INSTRUMENTAL EN EL ACTO DEL INFIERNO DE LA ÓPERA CÁSTOR Y PÓLUX DE RAMEAU}

Fred Büttner

Ludwig-Maximilians-Universität, München (Deutschland)

\begin{abstract}
Zusammenfassung:
In der neueren Musikgeschichte wird das Kontinuum des instrumentalen Spiels zunehmend aufgebrochen und in Diskontinuität verwandelt - ein Vorgang, der mit dem Schaffen der Wiener Klassik in einer kompositorischen Faktur gipfelt, in der die fortlaufende Spielbewegung im Taktsystem und deren abruptes Aufbrechen zu idealem Ausgleich gebracht werden. Eine wesentliche Station auf diesem Entwicklungsgang bildet, wie Thrasybulos Georgiades anschaulich gezeigt hat, die Musik der „Opera buffa“ als neuer Gattung in der ersten Hälfte des 18. Jahrhunderts, wie sie namentlich durch Pergolesis Serva padrona vertreten wird. Darüber sollte man jedoch den französischen Anteil nicht vernachlässigen, der sich in Rameaus „Tragédies en musique“ eindrucksvoll zu Wort meldet. Eine Betrachtung des Höllenakts aus Castor et Pollux macht deutlich, dass auch bei Rameau das instrumentale Spiel, obwohl es mit seiner zuweilen beunruhigenden Motorik noch einmal das alte Kontinuum der Instrumentalmusik realisieren kann, andererseits immer wieder von einer Diskontinuität erfasst wird, in der sich das aufregende Bühnengeschehen musikalisch niederschlägt.
\end{abstract}

\section{Schlüsselwörter:}

Air des Démons; Castor et Pollux; Diskontinuität; Höllenakt; Instrumentales Spiel; Lully; Pergolesi; Phébé; Rameau; Tragédie en musique.

\section{Abstract:*}

In modern Music History the continuity of instrumental playing gets increasingly broken up and changes into discontinuity - a process which, in the works of the Viennese Classic, culminates in a compositional mindset that brings the continuous instru-

* Ich danke Iwo Zaluski für seine Ratschläge bei der Abfassung des englischen „Abstract“. 
mental flow and its abrupt break-up to ideal balance. A significant stadium in this development is formed by the music of „Opera buffa" as new genre in the first half of the eighteenth century, as it is represented by Pergolesi's Serva padrona. On the other hand the French contribution, which makes itself felt with Rameau's „Tragédies en musique“, should not be neglected. A detailed study of the Hell Act from Castor et Pollux shows that also in the works of Rameau instrumental playing, even if it is able to realize, with its at times disquieting motivity, once again the old continuity of instrumental music, gets repeatedly stroken by discontinuity, in which the exciting actions on the stage find their musical expression.

\section{Key Words:}

Air des Démons; Castor et Pollux; Discontinuity; Hell Act; Instrumental Playing; Lully; Pergolesi; Phébé; Rameau; Tragédie en musique.

\section{Resumen:}

La continuidad de la música instrumental -en la moderna Historia de la Música-, experimenta un creciente proceso de fragmentación que la transforma en discontinuidad. Precisamente, este proceso halla su culminación en la escritura compositiva de las óperas de los clásicos vieneses, en donde el fluir continuo del discurso musical -articulado en un sistema de compases bien definido-, alcanza una relación ideal de equilibrio con sus respectivos momentos de pausa o interrupción. Un estadio importante en este desarrollo evolutivo -como ha demostrado Thrasybulos Georgiades- lo representa la música de la ópera bufa, como nuevo género de la primera mitad del siglo XVIII, tal y como se muestra en la Serva padrona de Pergolesi. No se debe desdeñar tampoco la contribución francesa, que emerge con todo su vigor expresivo en las tragédias en música de Rameau. El estudio detallado del Acto del infierno de la ópera Cástor y Pólux evidencia esta misma articulación del discurso instrumental en las obras de Rameau, aun dándose cuenta de que la antigua continuidad de la música instrumental (con su mecánica motora -en ocasiones, incluso inquietante-), se ve, por otro lado, frecuentemente afectada por una cierta discontinuidad, en la que la música refleja la trama de la escena, y construye los momentos de mayor dramatismo.

Palabras clave:

Aria de los demonios; Cástor y Pólux; discontinuidad; Acto del infierno; música instrumental; Lully; Pergolesi; Febe; Rameau; Tragedia en música.

Jean-Philippe Rameau gilt heute als einer der größten Opernkomponisten, die Frankreich je hervorgebracht hat, und ohne Zweifel als bedeutendster Vertreter der „Tragédie en musique“, d.h. der gesungenen Tragödie, im 18. Jahrhundert. Dies war nicht immer so. Seit der ersten Aufführung von Hippolyte et Aricie an der Pariser „Académie Royale de Musique“ vom 1. Oktober 1733 mussten sich Rameaus Werke den Vorwurf gefallen lassen, die Sprache und mit ihr das Bühnendrama durch Betonung des eigenständig Musikalischen zu überlagern und so die französische, von Jean-Baptiste Lully begründete Nationaltradition zugunsten italienischer Einflüsse aufzugeben.

Das stärkere Hervortreten des musikalischen Anteils, nach Überzeugung der „Lullisten“ das eigentliche Hauptargument gegen Rameau, konnte aber im Munde seiner Anhänger auch zu einem Qualitätsmerkmal werden, das ihm schließlich sogar mehr Bedeutung als Lully selbst einräumt. So veröffentlichte der Postillon françois im Juli 1739, wenige Monate vor der Erstaufführung von Rameaus dritter „Tragédie en musique” Dardanus, einen ausgesprochen ,ramistischen“ Artikel, der den Komponisten im Unterschied zu Lully für so genial erklärt, dass Wirkung und Rang seiner Opern auf die Teilhabe der Dichtung kaum mehr angewiesen seien ${ }^{1}$ :

1 Le Postillon françois, 1739: 26-38; folgendes Zitat: 34f. - Das genaue Erscheinungsdatum ist unbekannt. Der Artikel trägt die Überschrift „,De Paris ce 30 Juin 1739“, ihm folgt ein weiterer Artikel mit Datum vom 2. Juli. 
Lulli doit autant à Quinault qu'à son propre génie: Campra \& Destouches n'ont pas moins d'obligation à la Motte \& à Danchet: mais un aussi grand homme que Rameau, ne devoit briller que de son propre éclat; lui seul pouvoit se suffire à lui-même, \& suffire à tout le monde. La Poësie a son Théatre; qu'elle aille y déployer tous ses avantages! La Musique doit avoir aussi le sien: qu'on ne le lui dispute plus; qu'elle y regne seule; qu'elle en chasse toute rivale, qui veut lutter contre elle, \& qu'elle n'en admette qu'autant que leur difformité pourra lui servir de lustre. Principes dignes du Restaurateur de la belle harmonie, \& digne de ceux qui n'ont pû résister à tant d'attraits ravissans!

Eine solch strenge Definition von „Musiktheater“, derzufolge sich das theatralische Ereignis primär in der Komposition zu verwirklichen habe, die zu diesem Zweck alle ihre Mittel zum Einsatz bringen soll, wirft die Frage nach dem konkreten Verhältnis von Musik und Sprache in den Opernpartituren Rameaus auf, und zwar besonders dort, wo musikalische Strukturen augenscheinlich weniger im unmittelbaren Dienst des Sprachvortrags stehen, sondern einer eigenen Gestaltungskraft entspringen. Um diese Frage zu untersuchen, sei im folgenden der berühmte Höllenakt aus Castor et Pollux, Rameaus zweiter „Tragédie en musique“, näher betrachtet, dem schon nach seiner ersten Aufführung am 24. Oktober 1737 von Seiten des Opern- und Theaterliebhabers Simon Henri Dubuisson das Lob zuteil wurde, „le chef-d'œuvre des chefs-d'œuvre de musique“" zu sein².

Bevor wir uns jedoch dem musikalischen Satz zuwenden, scheint es nötig, den Inhalt des Höllenaktes zu skizzieren, weil nur so die Rolle der Musik innerhalb des theatralischen Gesamtverlaufs und namentlich ihre Beziehung zum Text überprüft werden können. Zu Beginn [III $]_{1}$ ruft die spartanische Prinzessin Phébé an der Höllenpforte das auf ihr Geheiß versammelte Volk und die noch fernbleibenden Höllengeister um Beistand an: Sie sollen verhindern, dass der von Jupiter gezeugte Pollux seinen gefährlichen Plan ausführt, in die Hölle hinabzusteigen und den im Kampf mit dem Nebenbuhler Lincée gefallenen Halbbruder, König Castor, ins Leben zurückzuholen. Dass Phébé so sehr um den Göttersohn besorgt ist, hat freilich einen tieferen Grund, denn sie ist schon lange in ihn verliebt. Jedoch wird ihre Liebe nicht erwidert, so dass auch ihr Versuch, ihn selbst von seinem Vorhaben abzubringen, ohne Erfolg bleibt $\left[\mathrm{III}_{2}\right]$. Schlimmer noch, muss sie im weiteren Gespräch mit Pollux und der soeben eintreffenden Télaïre erkennen, dass der Anstoß zur waghalsigen Unternehmung von jener, wie Pollux unsterblichen Sonnentochter ausgeht, die auf diesem Wege ihren Geliebten Castor wiederzugewinnen hofft [ $\left.\mathrm{III}_{3}\right]$. Dabei kommt ihr großer Einfluss auf Pollux nicht von ungefähr: Ohne Umschweife teilt der Göttersohn nunmehr der ahnungslosen Phébé mit, dass er Télaïre seinerseits liebe. Auf ihre Veranlassung steige er ins Totenreich hinab, um sie mit dem geliebten Bruder glücklich zu vereinen. Die heldenhafte Befreiungsaktion erweist sich damit als entsagungsvolles Opfer, da die Rückkehr Castors zugleich für Pollux den eigenen Verzicht auf Télaïre besiegelt.

Indem die beiden unglücklich Liebenden Pollux und Phébé am Schluss der Szene gemeinsam die Macht der Liebe anklagen, während Télä̈re die Möglichkeit ungetrübten Gewinns sieht, setzen alle drei Protagonisten zuletzt einen kontemplativen Ruhepunkt, der sich sofort als „Ruhe vor dem Sturm“ er-

2 DUBUISSON, [1882]: 390. 
weist, denn in diesem Augenblick dringen die von Phébé gerufenen Dämonen aus der Hölle hervor und leiten damit die zweite Akthälfte ein $\left[\mathrm{III}_{4}\right]$. Nach einem längeren Kampf gelingt es Pollux mit Hilfe des herabschwebenden Mercure, den Widerstand der Dämonen zu brechen und, vom Götterboten geleitet, die unterirdischen Regionen zu betreten. Ihrer Ohnmacht bewusst werdend, ergibt sich Phébé ganz dem Bösen, denn das Geständnis des Pollux hat ihren Gefühlen eine neue Richtung gegeben: diejenige der Rache $\left[\mathrm{III}_{5}\right]$. Sie träumt von Verbrechen, die sie selbst in ein dämonisches Wesen verwandeln, nachdem ihr nicht gelungen ist, die eigentlichen Höllendämonen zu ihrem Werkzeug zu machen.

Obwohl Phébés den gesamten Akt beendender Schlussvers „Et déja tout l`Enfer a passé dans mon cœur“, der an die Königin der Nacht und ihre berühmte Arie „Der Hölle Rache kocht in meinem Herzen“ aus Mozarts Zauberflöte denken lässt, neue dramaturgische Perspektiven für diese Figur eröffnet, tritt sie mit der letzten Szene des Höllenaktes, nachdem sie auch zuvor nur in einem Gespräch mit Télaïre $\left[\mathrm{I}_{2}\right]$ aufgetreten war, wieder in den Hintergrund. Lediglich ein Monolog zu Beginn des fünften Aktes gibt ihr noch einmal Gelegenheit, ihre dunklen Gefühle zur Schau zu stellen $\left[\mathrm{V}_{1}\right]$. Wenn wir anschließend von Pollux erfahren, dass sich Phébé auf ewig in die Schlünde der Hölle zurückgezogen hat $\left[\mathrm{V}_{6}\right]$, so ist sie damit zum einzigen Opfer der Liebe (,amour“) in Rameaus zweiter „Tragédie en musique“ geworden, während bei Pollux ja die Bruderliebe (,,amitié“), d.h. die Tugend (,vertu“), über den Egoismus der Leidenschaft gesiegt hat. Deshalb ist nach dem Höllenakt, der die destruktiven Kräfte des Bösen mit furchtbarer Gewalt zum Vorschein bringt, das weitgehende Ausblenden Phébés und mit ihr alles Dämonischen nur folgerichtig, damit sich das Ideal selbstloser Bruderliebe, dem auch Castor mit seinem Entschluss gehorcht, nach einem letzten Abschied von Télaïre ins Elysium zurückzukehren, als eigentliches Thema der Handlung vollenden kann.

Die Ereignisse des Höllenaktes aus Rameaus Castor et Pollux gehören also zur Sphäre des Wunderbaren, einer in den natürlichen Lebensraum des Menschen einbrechenden Realität, die in der französischen Operntheorie des 17. und 18. Jahrhunderts, als „Merveilleux“ bezeichnet, von entscheidender Bedeutung war. Jedoch geht es keineswegs um den „obersten Bereich des Wunderbaren“, die an das Göttlich-Erhabene gebundenen „Momente höchster Feierlichkeit“, für die Reinhold Hammerstein besondere Kompositionsmittel wie lang gehaltene Klänge und tiefe Lage nachweisen konnte ${ }^{3}$, sondern im Gegenteil um die schrecklichen Abgründe des Unterirdischen. In diesem Sinne prägt auch die Musik Rameaus völlig andere Eigenschaften als die von Hammerstein beschriebenen aus, denn sie ist von einer motorischen Rastlosigkeit bestimmt, die dem ganzen Höllenakt ein überraschend einheitliches Gesicht verleiht.

Um aber diese motorische Rastlosigkeit zu erzielen, ist der Komponist in besonderem Maße auf das kontinuierlich weiterlaufende, dem festen Taktprinzip verpflichtete Instrumentalspiel angewiesen, demgegenüber die rezitativische Sprachdeklamation zurückgedrängt wird. Nur an drei Stellen ist überhaupt von „Rezitativ“ zu sprechen: beim Gespräch Phébé-Pollux, zu dem später noch Télaïre hinzutritt, bei der Schlussszene mit dem Monolog der Phébé und beim Mittelteil des Eröffnungssatzes, in dem Phébé die Dämonen zu Hilfe ruft. Doch bereits im ersten Fall gelingt es Phébé gerade einmal, dem auf der Szene

3 HAMMERSTEIN, 1995: 222-237. 
erscheinenden Pollux die Frage „Ah! Prince, où courez-vous?“ zu stellen, bevor er in sein Air ,Je vole à la victoire“ ausbricht, und Télä̈re wendet sich sofort mit einer eindrucksvollen Erzählung an Pollux, die man aufgrund ihrer reichen Verwendung des Orchesters dem „récitatif accompagné“ zurechnen muss. Immerhin verbleibt der lange Hauptabschnitt des Gesprächs auf der Ebene des „récitatif simple“, während Phébés Monolog am Ende des Höllenaktes schon nach wenigen Takten in ein „récitatif accompagné“ übergeht, dessen wütende Akkordbrechungen, wenn auch abwärts gerichtet, eine Verwandtschaft mit den hochschnellenden Blitzen in Télä̈res Erzählung aufweisen. Von ähnlichen Gestalten ist der Mittelteil des Eröffnungssatzes bestimmt, dessen musikalischer Charakter sich damit abermals im Orchester entscheidet.

So lassen bereits die wenigen Rezitativstellen des zentralen Höllenaktes eine Tendenz zur Vereinheitlichung erkennen, deren Auswirkungen bis in die Rahmenakte der Oper reichen. Der düstere Jubelchor der Spartaner angesichts des Todes von Castors Nebenbuhler, „Que l'Enfer applaudisse“, lässt mit seinem gleichförmigen Viertelkontinuum, in das die raschen Dreiklangsbrechungen der Achtel wie ein Bild zusammenschlagender Hände eingeblendet werden, schon im ersten Akt etwas von der unheimlichen Mechanik der Höllenmusik im dritten Akt ahnen $\left[\mathrm{I}_{4}\right]$, und wenn Pollux am Ende des dortigen Gesprächs mit Phébé und Télaïre auf das Erscheinen der Dämonen aufmerksam macht, so erinnert nicht nur seine Ausdrucksweise (besonders das Verb ,pâlir“) an den Beginn von Télaïres dem Chorsatz vorausgehender Totenklage „Tristes Apprêts, pâles flambeaux“ $\left[\mathrm{I}_{3}\right]$, auch der in zwei Hälften geteilte Abstieg von der höheren Lage des Grundtons $a$ über dessen Unterquint $d$ zur tieferen Lage des Grundtons $A$ scheint dem kargen, lediglich aus den Tönen $e s^{2}-a s^{l}$-es ${ }^{l}$ bestehenden Melodiegerüst der Totenklage nachgebildet. Im fünften Akt schließlich, wenn Castor und Télä̈re angesichts einer plötzlich aufgebrachten Natur noch einmal fürchten, der Hölle zum Opfer zu fallen, arbeitet die Komposition mit musikalischen Mitteln, die aus dem Höllenakt selbst bekannt sind: Rasche Sechzehntel verbinden sich zu Dreiklangsbrechungen, Läufen oder Tonrepetitionen $\left[\mathrm{V}_{4}\right]$. Diesmal jedoch kündigt das Unwetter nicht das Erscheinen der Dämonen, sondern das Nahen des rettenden „Deus ex machina“ an, und so weichen die Sechzehntel einer lieblichen Flötenmusik - ein Kontrast, der unwillkürlich den Eindruck entstehen lässt, als müsse sich der furchtbare Höllenschlund abermals vor den Freuden des Elysiums verschließen, wie es bereits im Wechsel zwischen Höllenakt und folgendem Elysiumsakt geschah.

Besonders wirkt sich die Fähigkeit der Musik zur Vereinheitlichung aber im Höllenakt selbst aus, und dies namentlich im instrumentalen Spiel der Ensemblesätze, welche, von Rezitativen kaum unterbrochen, zumeist durchgängig aneinander gereiht sind. Darum stellen auch die energischen Dreiklangsbrechungen im Mittelteil des Eröffnungssatzes, ungeachtet ihrer Nähe zu Télä̈res Erzählung und Phébés Monolog, in erster Linie den Zusammenhang mit dem Rahmenteil her, dessen als „Prélude“ bezeichnetes Vorspiel sofort mit einer in Vierteln abwärts zielenden Dreiklangsbrechung beginnt (Beispiel 1, Zeile 1), die im weiteren Satzverlauf, Phébés insistierenden Rufen und ihrer mehrmaligen Beantwortung durch die getreuen Spartaner entsprechend, hartnäckig wiederholt wird. Dabei legen ebenso das gleichförmige Viertelkontinuum wie der über eine Duodezim reichende Ambitus die Vermutung nahe, dass wir es mit einer musikalischen Gestalt zu tun haben, der sich die Sprache wohl zugesellen kann, die im Grunde aber nicht sprachgezeugt ist. 

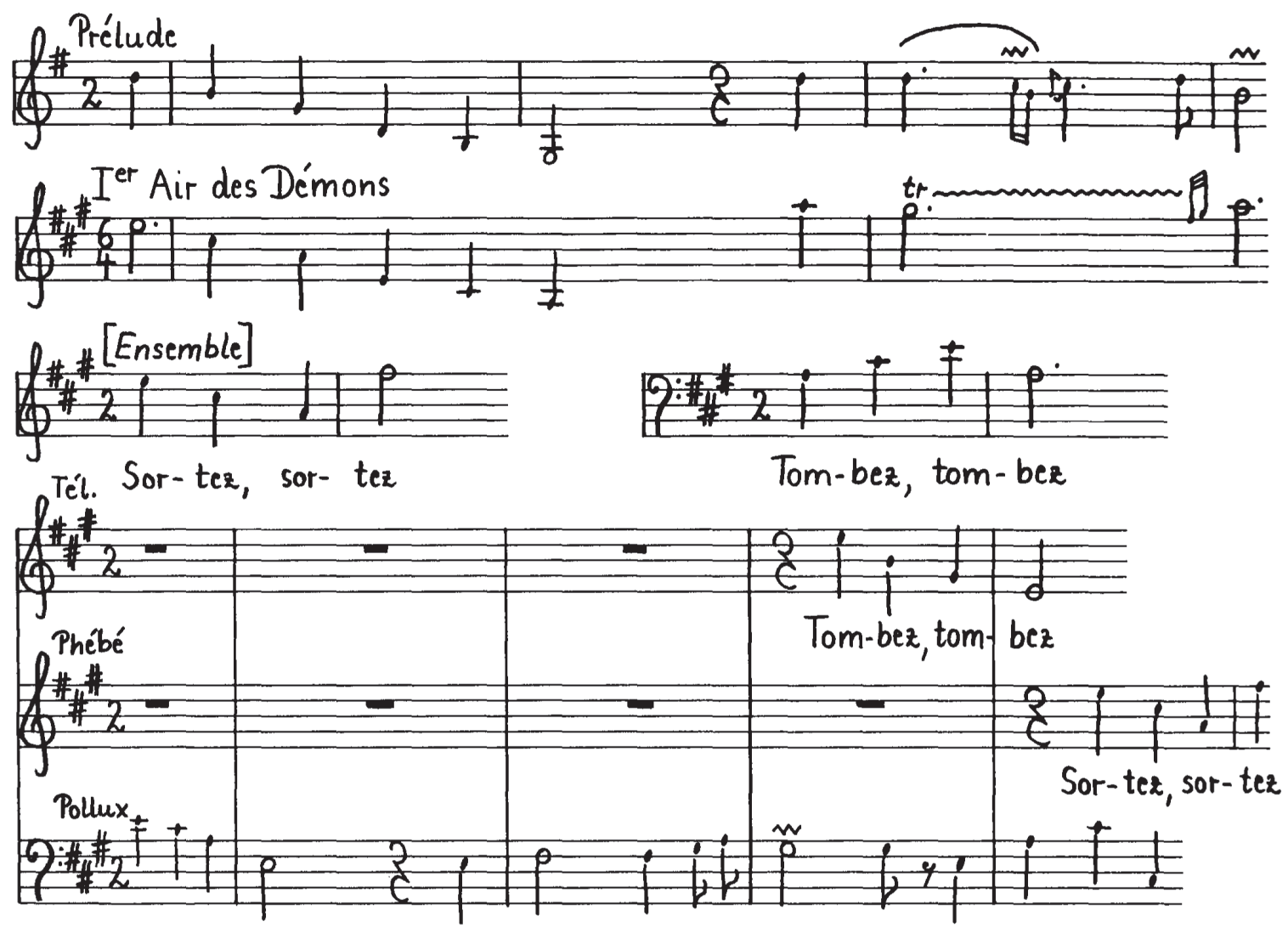

Tombez, tombez, ren-trez dansl'esclava- ge, Tom-bez, tom-bez

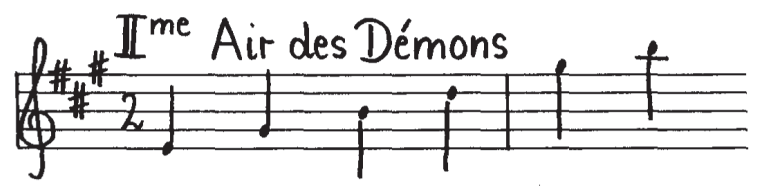

Die instrumentale Natur des in Vierteln nach unten steigenden Vorgangs wird durch die Neigung der Sänger unterstrichen, ihn auf einen dem Umfang der menschlichen Stimme angemessenen Ambitus zu verkleinern. So schiebt Phébé, als sie sich der Viertel auf die Worte „Des portes des Enfers“ bedient (Takt 21-28), den unteren Dreiklang um einen Ganzton nach oben, so dass die Duodezim zu einer Undezim wird. In der Antwort des Spartanerchors bringt es der führende „Dessus“, nachdem die Gestalt von den einleitenden „Basses“ in ihrer originalen Form benützt worden war, in zwei Schritten sogar auf eine Verkleinerung von der Septe bis zur verminderten Quint, bevor sich im abschließenden Viertakter (Takt 41-44) noch einmal die ursprünglichen Intervalle durchsetzen. 
In noch stärkerem Maße wirkt sich die Tendenz zum Verkleinern der Dreiklangsbrechung im großen Ensemblesatz aus, der das Zentrum des Höllenaktes bildet. Von den sechs Tönen der originalen Form bleiben nur drei bzw. vier Töne übrig, womit sich der Umfang auf eine Quint oder Oktav beschränkt (Beispiel 1, Zeile 3-4). Die eigentliche Antriebsfeder des Satzes ist aber auch hier das Instrumentalspiel mit seinen kontinuierlich weiterlaufenden Vierteln (,Basse continue“) und Achteln (übrige Streicher). Gleichzeitig werden die Taktgruppierungen einem dauernden Wechsel unterworfen, dessen gefährliche, dem Kampf des Pollux mit den Dämonen angemessene Unberechenbarkeit den Zuhörer in höchste Spannung versetzt und somit in das aufregende Bühnengeschehen hineinzieht.

Dass die auf gleichmäßige Viertel verteilte Dreiklangsbrechung im instrumentalen Spiel beheimatet ist, zeigt auch ihre Wiederkehr in den beiden „Airs des Démons“, zwei den Dämonenchor „Brisons tous nos fers“ umrahmenden Orchesterstücken. Dabei behält sie am Anfang des ersten Satzes nicht nur die vom Beginn des Aktes geläufige Intervallstruktur, sondern fügt dem Abstieg, ebenso wie dort, einen kadenzierenden Anhang hinzu (Beispiel 1, Zeile 2). Lediglich das rhythmische Profil scheint gegenüber dem Eröffnungssatz verzerrt, indem der Vorgang mit langem Auftakt $e^{2}$ in eine Dreierbewegung umgeformt wird. Auf diese Weise nimmt er einen tänzerischen Charakter an, wie es der Ballettsituation im „Air“ entspricht. Darüber hinaus lassen die Längung der Anfangsnote und der exponierte Neuansatz nach dem Abstieg bereits eine Zunahme der insgesamt im Höllenakt angelegten Tendenz für die „Airs des Démons“ erkennen, gängige Melodiegestalten zugunsten eines abgehackten Duktus mit großen, unvermittelten Sprüngen aufzugeben. Unsere Vorstellung von „Melodie“ als einem sanglichen, in sich schlüssigen Gesamtverlauf, der von menschlicher Empfindung getragen ist, wird außer Kraft gesetzt. An seine Stelle tritt ein seelenloser Mechanismus, der auf den Menschen bedrohlich wirkt.

Diese von den „Airs des Démons“ bis zum Grotesken gesteigerte Ausstrahlung wird dadurch weiter intensiviert, dass in das gleich einem Uhrwerk präzis ablaufende Spiel der Instrumente, wie wir schon im Zusammenhang mit dem zentralen Ensemblesatz bemerkt haben, trotzdem auch ein unberechenbares Moment eingelagert ist. So bringt es der Anfangsteil des ersten „Air“, obwohl sich dort Taktpaare herausbilden, auf nur fünfzehn Takte (der letzte vor dem Doppelstrich stehende Takt fädelt bereits wieder den Beginn ein). Jedoch liegt der Grund dafür weniger im taktweisen Einblenden zweier Achtelbewegungen, die im übrigen Verlauf des Anfangsteils fehlen (Takt 5 und 12). Die irreguläre Ausdehnung hängt vielmehr damit zusammen, dass es angesichts der kontrapunktischen Verzahnung der beiden Außenstimmen schon bald keine Rolle mehr spielt, ob man die auf- und absteigenden Viertel des einen oder die weit voneinander entfernten Einzeltöne des anderen Taktes als Beginn des Taktpaares bewertet - eine Destabilisierung der musikalischen Ordnung also, zu der freilich auch die eingeblendeten Takte das ihrige beitragen. Diese Ambivalenz erlaubt es dem Komponisten am Ende, die Viertelbewegung aus Takt 13 im folgenden Takt zu wiederholen und damit endgültig auf die geradzahlige Taktposition zu schieben, wodurch sich die alternierenden Einzeltöne auf den ungeradzahligen Takt 15 verlagern.

Auch im zweiten „Air des Démons“ haben wir mit beiden Dimensionen der musikalischen Motorik zu rechnen: dem monotonen Kontinuum des taktgebundenen Spiels und unerwartet eintretenden Brüchen. Dabei lässt die Verteilung der im gesamten Höllenakt gegenwärtigen Viertel auf zwei Takte erkennen, 
dass dem Satz auch diesmal eine Gliederung in Taktpaaren zugrunde liegt (Beispiel 1, Zeile 5). Jedoch enthält der Anfangsteil wieder eine ungerade Zahl von Takten, und wir haben, wie beim ersten „Air“, nach der Ursache zu forschen. In diesem Zusammenhang scheint es wesentlich, dass der Dreiklangsbrechung in Vierteln jetzt eine andere, ebenfalls zweitaktige Gestalt gegenübertritt, die von einem flüssigeren Achtellauf eingeleitet wird, um danach pointiert mit einem abgesetzten Dreitonelement zu schließen (Beispiel 2, Zeile 2). Die irreguläre Ausdehnung des Anfangsteils kommt nun aber dadurch zustande, dass sich die Achtel mit ihrer genuin instrumentalen Reihung viertöniger Formeln unmittelbar nach dem ersten Auftreten der zweitaktigen Gestalt am Beginn (Takt 1f.) zu einem taktweise sequenzierenden Abstieg verselbständigen, der eine erweiterte Phase von insgesamt drei Takten bewirkt. So ist diese Unregelmäßigkeit erneut in der gespannten Dynamik des Spielvorgangs begründet, der offenbar unter dem Einfluss choreographischer Vorstellungen steht.
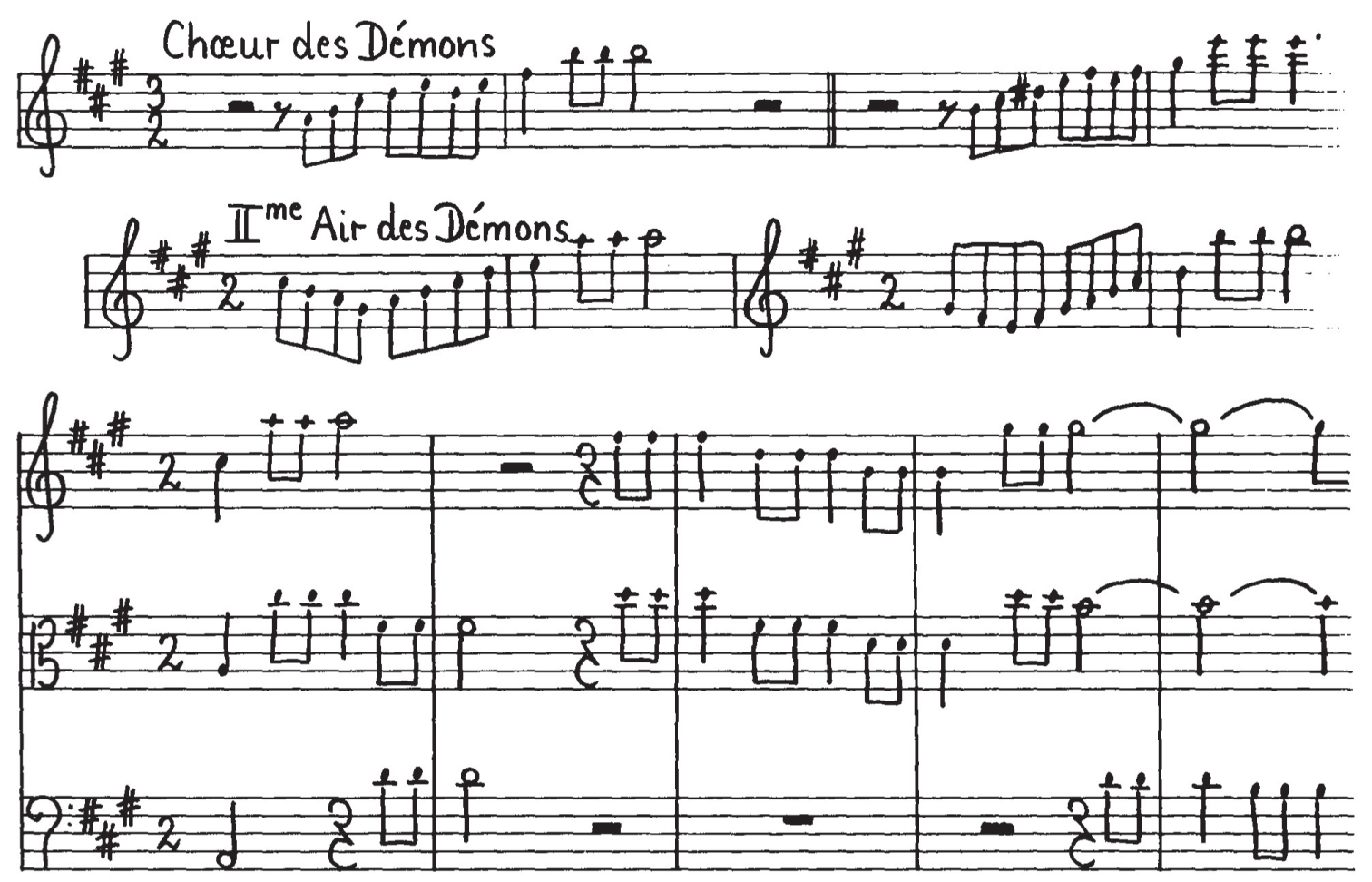

Es zeigt sich also, dass in Rameaus Höllenakt ein enger Zusammenhang von kompositorischer Faktur und Bühnengeschehen existiert, der zum einen für die rastlos vorwärts treibende Kraft des instrumentalen Satzes, zum anderen aber auch für den abrupten Wechsel musikalischer Situationen und das gleichzeitige Gegenüber unterschiedlicher Gestalten verantwortlich ist. Behält man diesen Zusammenhang im Auge, so versteht man, warum nach der Einführung des zweitaktigen Elements am Anfang des zweiten 
„Air des Démons“ (Takt 1f.) und der dreitaktigen Fortspinnung seiner viertönigen Achteleinheiten (Takt 3-5), die zugleich den Umschlag von einem aufwärts zu einem abwärts gerichteten Vorgang realisiert, in den Violinen unvermittelt die nun energisch emporsteigende Dreiklangsbrechung früherer Sätze wiederkehrt, während sich die Bassstimme noch einmal der vorausgehenden Sequenzfigur bedient und diese ihrerseits sequenzierend zum Grundton $A$ weiterführt. Ebenso versteht man jedoch die Bedeutung, welche bereits im Dämonenchor „Brisons tous nos fers“ dem plötzlichen Hineinfahren des zweitaktigen Elements (Beispiel 2, Zeile 1) und einer eintaktigen, an den Chorsatz „Que l'Enfer applaudisse“ erinnernden Dreiklangsbrechung, wiederum auf Achtelebene, in das gleichförmige Raster aus Viertel- und Achtelschlägen zukommt: Indem die Instrumentaleinwürfe das vorhersehbar scheinende, in Wahrheit aber aus unterschiedlich langen Taktgruppen gebildete Kontinuum stören, lassen sie umso mehr die unberechenbare Wildheit der höllischen Geister spüren, deren gefährliche, dem Menschen feindlich gesinnte Natur betont wird.

Diese für den Höllenakt bezeichnende Diskontinuität der kompositorischen Faktur erreicht ihren Gipfelpunkt im zweiten Abschnitt des dem Dämonenchor folgenden „Air“, d.h. kurz bevor Pollux den Kampf zu seinen Gunsten entscheidet. Hier spaltet sich das Dreitonelement der zweitaktigen Gestalt im fünften Takt von seinem Vorlauf ab und steht als separater, eine Generalpause nach sich ziehender Rhythmus vor uns (Beispiel 2, Zeile 3). Spätestens hier wird deutlich, dass die unerwarteten Brüche von Rameaus Musik im Extremfall eine zerstörerische Gewalt entwickeln können, die den rhythmischmetrischen Fluss ernsthaft gefährdet. Und so kehrt die Komposition nach diesem Ereignis auch nicht sofort zu ihren längeren Achtelketten zurück, sondern beißt sich gewissermaßen am dreitönigen Impuls fest, der insgesamt noch sechsmal wiederholt wird, bevor eine Kadenzwendung in die jetzt aufwärts sequenzierende Imitation der dreitaktigen Fortspinnung aus dem Anfangsteil führt. Aber selbst dieser, dem melodischen Profil der zweitaktigen Gestalt vom Satzbeginn treu bleibende Vorgang wird durch Ergänzung der Dreitongruppe im vierten Takt aus seiner Bahn geworfen, so dass die Bewegung danach wiederum neu ansetzen muss.

Konfrontiert uns Rameaus Musik mit einer derart ausgeprägten Diskontinuität, so können wir nicht umhin, dabei an die herausragende Bedeutung zu denken, die einer diskontinuierlichen Satzfaktur nach Thrasybulos Georgiades bei den Wiener Klassikern zukommt ${ }^{4}$. Findet die Diskontinuität auch dort, wie bei Rameau, primär im instrumentalen Spiel statt, so bringt sie Georgiades dennoch mit aktivem Sprachvollzug in Verbindung, der auf musikalischer Ebene, wie er exemplarisch am Beispiel von Pergolesis Serva padrona (1733) veranschaulichte, erstmals durch die italienische „Opera buffa“ realisiert wird. Von der Schwere eines kunstvoll ausgearbeiteten Satzes befreit, gelinge es der „Opera buffa“ mehr als früheren Gattungen der Musikgeschichte, das „Hier und Jetzt“ der menschlichen Wirklichkeit unmittelbar zu erfassen.

Nun bewegen wir uns mit Castor et Pollux zwar in der zeitlichen Nähe von Pergolesis „Opera buffa“, andererseits jedoch statt auf italienischem auf französischem Boden. Und selbst wenn die Zeit-

4 GEORGIADES, 1951: 77-80. Wiederabdruck in GEORGIADES, 1977: 10-13. 
genossen Rameaus seinen „Tragédies en musique“ von Anfang an eine Nachbarschaft zur italienischen Oper nachsagten, so meinten sie kaum die neue Gattung der „Opera buffa“, sondern die etablierte „Opera seria“. Trotzdem ist im reichen Instrumentalspiel des zentralen Höllenaktes aus Castor et Pollux etwas vom scharfen Erfassen des Augenblicks und damit eine der Wiener Klassik verwandte Haltung zu spüren - und das, obwohl es nach unseren Überlegungen keineswegs scheinen will, als sei das Plötzliche, Überraschende in Rameaus Musik von sprachlicher Substanz angeregt.

Im Gegenteil bringt uns die bisherige Analyse der Beziehung von Vokal und Instrumental während des Höllenaktes zu der Schlussfolgerung, dass die Diskontinuität bei Rameau im instrumentalen Spiel selbst verwurzelt ist. So erscheint die erste der beiden musikalischen Gestalten, die wir näher untersucht haben (Beispiel 1), zwar auch im Gesang, doch verwiesen ihre gleichförmigen Viertel und der ungewöhnlich große Ambitus auf eine Herkunft im Orchestersatz, wo sie ja zu Beginn des Höllenaktes zuerst verwendet wird. In diesem Sinne beschränkt sich die zweite musikalische Gestalt (Beispiel 2) nun tatsächlich auf die Instrumente, und wenn sie im Dämonenchor gleichzeitig mit Sprache erklingt, so bildet sie ihr gegenüber eine eigene Schicht, die sich wie ein Störfaktor in das Kontinuum einzuschalten versucht.

Und doch scheint es gerade bei dieser sich nirgendwo mit Sprachsilben verbindenden Gestalt eine innere Beziehung zur sprachlichen Sphäre zu geben. In ihrem dreitönigen Impuls, der durch Trennung von den vorausgehenden Achteln auf Diskontinuität angelegt ist, begegnet uns derselbe Rhythmus, den wir im Rahmen der Dichtung als „Anapäst“ kennen. Dort spielt er aber, neben dem Jambus, namentlich im französischen Alexandriner eine entscheidende Rolle, dem hauptsächlichen Vers nicht nur der gesprochenen „Tragédie“, sondern auch der „Tragédie en musique“. Aus diesem Grunde ist es offenbar kein Zufall, wenn der dreitönige Impuls bei seiner Verselbständigung im zweiten „Air des Démons“ (Beispiel 2, Zeile 3) auch innerhalb des instrumentalen Spiels viermal nacheinander von der Oberstimme eingesetzt wird, was genau dem zwölfsilbigen Gerüst des Alexandriners entspricht. Die Vermutung drängt sich auf, Rameau habe als französischer Komponist den Alexandriner im täglichen Umgang so verinnerlicht, dass er sich von der Sprachdeklamation in das instrumentale Spiel fortpflanzen konnte.

Eine solche Annahme wird besonders von zwei, in unterschiedlichem Grade rezitativischen Passagen des Höllenaktes unterstützt: einerseits Phébés Beschwörung der Dämonen im Mittelteil des Eröffnungssatzes, andererseits dem als ,récitatif accompagné“ gearbeiteten Schluss ihres Monologs am Aktende. In beiden Fällen besteht der vorzutragende Text überwiegend aus gelegentlich jambischen, meist aber anapästischen Alexandrinern, die bei der musikalischen Umsetzung normalerweise ihren Versrhythmus beibehalten. Nur wenn es bereits auf der sprachlichen Ebene zu individuellen, mit dem rhythmischen Schema des Verses nicht übereinstimmenden Akzenten kommt, gibt auch die Komposition den neuen Betonungen nach. Dasselbe gilt für die an mehreren Stellen eingeschobenen Acht- oder Zehnsilber.

Obwohl beide Passagen wesentlich vom Sprachvortrag reguliert werden, lässt sich daneben auch eine dauernde Interaktion von Gesangstimme und Orchester beobachten. So ist der gesamte Mittelteil des Eröffnungssatzes mit dreitönigen Unisono-Elementen der Streicher durchsetzt, welche die anapästischen Rhythmen der Sprache mitvollziehen oder ihnen gegenübertreten. An der entscheidenden Stelle aber, wenn der Befehl „Monstres, déchainez-vous“ den Alexandriner ganz aus den Angeln hebt und in zwei 
Hälften mit jeweils gedehnter Anfangsnote und dicht gedrängten Achteln aufspaltet, kommt es in den Streichern abermals, wie im zweiten „Air des Démons“, zu einem Komplex von vier dreitönigen Impulsen, der sich gleichsam als instrumentaler Alexandriner zwischen die Vershälften schiebt.
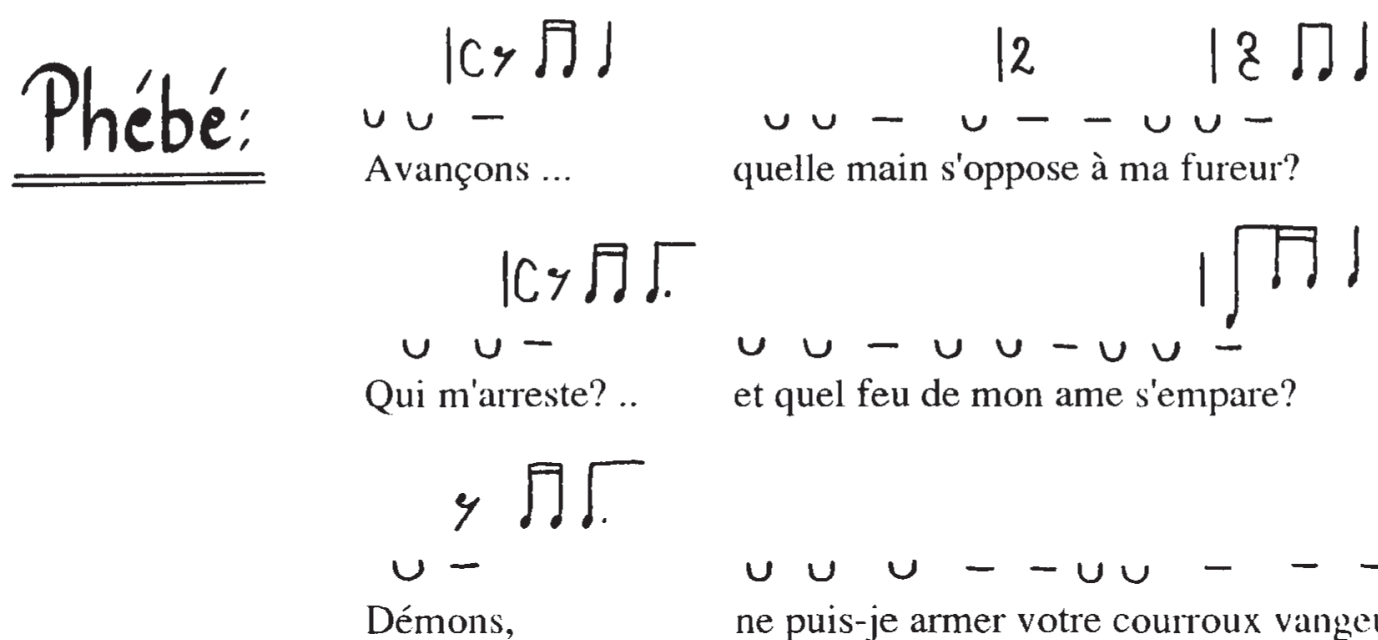

Démons, $u \cup u--u v--$ ne puis-je armer votre courroux vangeur?

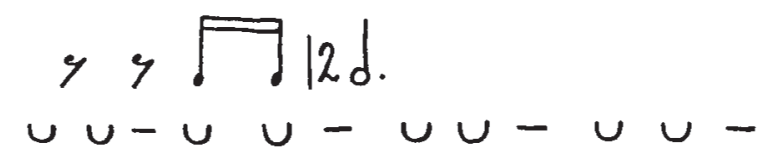

Pour aller jusqu'a vous s'il ne faut que dés crimes,

$-\quad \cup \cup-\quad \cup \cup-\cup \cup-$
Mon désespoir m'ouvrira vos abimes,

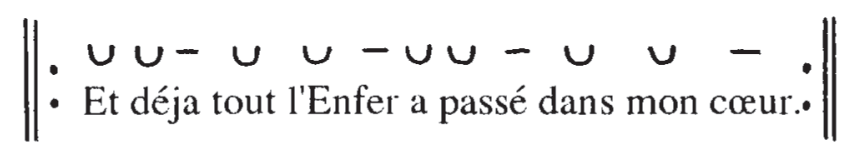

DIE ZEICHEN - UND U BEZIEHEN SICH AUF DIE MUSIKALISCHE BEHANDLUNG DES TEXTES

Der zweite Abschnitt von Phébés Monolog am Ende des Höllenaktes bildet dann mit seinem Wechsel von Gesangstimme und Orchester in den drei ersten Alexandrinern sogar eine feste Struktur aus (Beispiel 3): Zuerst wird ein im ersten und zweiten Vers anapästisches, im dritten Vers jambisches Kurzglied von den folgenden Silben durch einen dreitönigen, wiederum anapästischen Einwurf des Orchesters ge- 
trennt, anschließend unterstreicht der dreitönige Einwurf beim ersten und zweiten Alexandriner auch das Versende, während dem dritten Alexandriner eine vehemente Dreiklangsbrechung nach unten folgt, die schon zur letzten Versgruppe überleitet. Jedoch nimmt das Orchester auch in dieser Schlussphase am nun durchgehend anapästischen Rhythmus der Gesangstimme teil, wenn es zu Beginn deren zielgerichtetes „Pour aller jusqu'à vous“ mitvollzieht.

Deuten diese Beobachtungen klar auf den Zusammenhang des instrumentalen Spiels mit der Sprachdeklamation, so ist dennoch davor zu warnen, die in den Orchestersatz einbrechende Diskontinuität undifferenziert nur aus sprachlichen Vorbildern abzuleiten. Zumindest ist das Versschema des Alexandriners, dem der anapästische Rhythmus der Instrumente entspringen dürfte, insgesamt nicht auf Diskontinuität, sondern auf einen regelmäßigen Vortrag angelegt. Aber auch das instrumentale Spiel selbst, wie es seit dem späten Mittelalter in praktischen Quellen greifbar ist, tendiert von Anfang an zu einem kontinuierlichen Weiterfluss; das ereignishafte „Hier und Jetzt“ ist ihm wesensfremd.

Suchen wir nach dem auslösenden Moment für das Diskontinuierliche in Rameaus Castor et Pollux, und namentlich dessen Höllenakt, so dürfen wir zuerst weder an Musik, noch an Sprache denken. Das Gliedern in unterschiedlich lange Taktgruppen, die jäh in den Satzverlauf hineinfahrenden Einwürfe und seine unvermittelten Brüche hängen vielmehr mit der gleichzeitigen Aktion auf der Bühne, den dramatischen, ja entsetzlichen Ereignissen an der Höllenpforte zusammen. Die Sprache ist hierbei insofern einbezogen, als sie dem auf die Wirklichkeit reagierenden und sie mitgestaltenden Menschen angehört. In diesem Sinne hat auch Pergolesi - und mit ihm Georgiades - den italienischen Ottonario „Aspettare, e non venire“ zu Beginn der Serva padrona nicht primär als Vers, sondern als Prosa verstanden.

Die Fähigkeit, den Augenblick mit Hilfe der musikalisch umgesetzten Sprache zu erfassen, zeigt sich nun, trotz der stets spürbaren Wirkkraft des französischen Verses, zweifellos auch in Rameaus Umgang mit dem Castor et Pollux-Text. Dass sich dann jedoch der Orchestersatz in entscheidender Weise am Erfassen des Augenblicks beteiligt, das ist tatsächlich ein bemerkenswertes Faktum, das nicht weniger unmittelbar als Pergolesis Vorgehensweise, die der Sprache unbedingten Vorrang einräumt und im Gegensatz zu Rameau den instrumentalen Apparat nicht beibehält oder gar anreichert, sondern reduziert, die musikalische Zukunft der Wiener Klassiker ahnen lässt.

So begegnet uns in Jean-Philippe Rameau ein Opernkomponist, der die französische, durch Lully begründete Nationaltradition gewiss nicht zugunsten italienischer Einflüsse aufgab, wie es ihm manche Gegner unterstellten, dem es aber gelang, eine tiefe, durch eigene Äußerungen mehrmals unterstrichene Verwurzelung in dieser Tradition mit musikalischen Möglichkeiten der italienischen Oper zu verbinden. Dabei beschränkte sich die Offenheit gegenüber Italien nicht nur auf die etablierte Gattung der „Opera seria“: In einem musikalischen Satz, der mit hoher Sensibilität am Bühnengeschehen und dem Schicksal der darin involvierten Figuren teilnimmt, wird zumindest eine geistige Verwandtschaft mit den neuesten, in der Gattung der „Opera buffa“ konzentrierten Strömungen der italienischen Musik erkennbar, wenn auch die angewendeten Mittel hier in eine volle Orchesterbesetzung eingebunden bleiben.

Dass ein wacher, zudem auf dem Gebiet der Musiktheorie und musikästhetischen Diskussion hervortretender Komponist wie Rameau keine Bemühungen unternahm, sein Opernschaffen in den europäischen 
Kontext der eigenen Lebenszeit zu stellen, möchte man, wohl zu Recht, für unwahrscheinlich halten. Aus diesem Grunde dürfen wir damit rechnen, dass Rameau die aktuellen Tendenzen in Italien sehr genau beobachtete und sich selbst zu ihnen in Beziehung setzte. Ob ihn dabei wirklich das Gefühl überkam, er werde eines Tages „durch italiänische Virtuosen [...] begraben“ werden, was ihn angeblich „,mißmüthig, traurig und ärgerlich“"stimmte, wie Denis Diderot in seinem Dialog Rameaus Neffe behauptet ${ }^{5}$, sei dahingestellt. Andererseits belegt eine Äußerung gegenüber dem Schriftsteller François Arnaud, die uns André-ErnestModeste Grétry in seinen Mémoires, ou Essais sur la musique mitteilt ${ }^{6}$, dass er die zukunftweisenden Perspektiven der italienischen Musik scharfsichtig erkannte, wenn es auch unklar bleibt, wie wörtlich wir seine im selben Atemzug offen zur Schau getragene Bescheidenheit zu nehmen haben:

Si j'avois trente ans de moins [...], j'irois en Italie, Pergolèze seroit mon modèle; j'assujettirois mon harmonie à cette vérité de déclamation qui doit être le seul guide des musiciens; mais à soixante ans, 1 'on sent qu'il faut rester ce que l'on est. L'expérience dit assez ce qu'il faudroit faire; mais le génie refuse d'obéir.

Rameaus Worte vermitteln deutlich seine Überzeugung, dass sich die Musik am Sprachvollzug orientieren solle. Obwohl er gleichzeitig den Eindruck zu erwecken versucht, sein Alter (die Äußerung stammt etwa von 1743, also nur wenige Jahre nach der Komposition von Castor et Pollux) mache es ihm unmöglich, diese durch die zeitgenössische Musik Italiens vermittelte Einsicht noch im eigenen Schaffen zu realisieren, haben unsere Betrachtungen gezeigt, dass es die geforderte Orientierung der musikalischen Faktur am gesprochenen bzw. gesungenen Wort bei Rameau tatsächlich gibt. Indem sie sich aber mit einer auffälligen Diskontinuität im instrumentalen Spiel verbindet, sind seine „Tragédies en musique“ in hohem Maße an der Vorbereitung der musikgeschichtlichen Zukunft beteiligt. Aus der im Werden begriffenen Fähigkeit der Instrumentalmusik, nicht nur am aktiven Sprachvollzug, sondern mit ihm und über ihn am „Hier und Jetzt“ des menschlichen Lebens teilzuhaben, alle Konsequenzen zu ziehen, sollte freilich einer späteren Zeit vorbehalten bleiben.

\section{BIBLIOGRAPHIE}

DIDEROT, Denis, Rameau's Neffe. Ein Dialog von Diderot, aus dem Manuskript übersetzt und mit Anmerkungen begleitet von Goethe, Leipzig, Göschen, 1805.

DUBUISSON, Simon Henri, Lettres du Commissaire Dubuisson au Marquis de Caumont 1735-1741, ROUXEL, Albert (Hrsg.), Paris, P. Arnould, [1882].

GEORGIADES, Thrasybulos, „Aus der Musiksprache des Mozart-Theaters“, Mozart-Jahrbuch 1950, Internationale Stiftung Mozarteum (Hrsg.), Salzburg, Internationale Stiftung Mozarteum, 1951: 76-

5 DIDEROT, 1805: 15.

6 GRÉTRY, 1797: Bd. I, $426 f$. 
98. Wiederabdruck in GEORGIADES, Thrasybulos, Kleine Schriften = Münchner Veröffentlichungen zur Musikgeschichte, 26, Tutzing, Hans Schneider, 1977: 9-32.

GRÉTRY, André-Ernest-Modeste, Mémoires, ou Essais sur la musique, 3 Bände, zweite Auflage, Paris, Imprimerie de la République, 1797.

HAMMERSTEIN, Reinhold, „Invokation - Götterspruch - Orakel: Zur Topik des Wunderbaren in Bühnenwerken von J. Ph. Rameau“, Studien zur Musikgeschichte: Eine Festschrift für Ludwig Finscher, LAUBENTHAL, Annegrit (Hrsg.), Kassel, Bärenreiter, 1995: 222-237.

Le Postillon françois. Second Courrier, Brüssel, 1739: 26-38.

Recibido: 12/12/2012

Aceptado: 21/02/2013 